\title{
A graphical installation system for the GNU/Linux Debian distribution
}

\author{
Fiandrotti Attilio ${ }^{1}$,Pierluigi Di Nunzio ${ }^{1}$, Federico Di Gregorio ${ }^{1}$,Angelo Raffaele Meo ${ }^{1}$ \\ 1 Politecnico di Torino, Dipartimento Automatica e Informatica, \\ Centro Primario di Competenza sul Software Libero \\ Corso Duca degli Abruzzi 24, Torino, Italy \\ fiandro@initd.org, pierluigi.dinunzio@polito.it, \\ fog@debian.org, meo@polito.it \\ WWW home page: http://freesoftware.polito.it/
}

\begin{abstract}
One of the main objectives of the Centro di Competenza sul Software Libero del Politecnico di Torino is to provide custom GNU/Linux distribution to the Public Administration, small and medium enterprise and schools. Debian GNU/Linux was choosen as the base for the custom distributions because of its strong support of free software and its long-standing technical merits: minimalist hardware requirement, the best available packaging system, support for 13 different architectures and a strict set of quality guidelines adopted by all the active Debian developers. The only foreseeable limitation, the Debian default text-based installer, was overriden by restarting the development of the then-abandoned Debian graphical installer. Now the new graphical installer is developed by tens of people and it will be included in the next official Debian release.
\end{abstract}

\section{Custom Debian distributions}

One of the main objectives of the Centro di Competenza sul Software Libero del Politecnico di Torino is to provide custom GNU/Linux distributions to the PA and educational world; such distributions will be preconfigured and easily installable to minimize the amount of manual operations required to the final user.

Debian GNU/Linux was deemed to be the perfect distribution to base our custom work on, mainly because of its strong support of free software (as in the Debian Free Software Guidelines), its support for multiple hardware architectures, robust package management system and flexible and easily customizable installation system.

In particular, we needed an installation system with the following characteristics:

1. Released (and releasable) under a free software license (Debian does);

2. With a strong developers base (Debian does);

3. Modular and independent on packages about to be installed (Debian does);

4. Pre-configurable, to reduce the steps needed to install a working system (Debian does);

5. With a state-of-the-art User Interface supporting non-latin wide-char alphabets and a variety of input/output devices.

The only limitation to use the default Debian installer was point (5): when the project was started the Debian installer only supported a text-based front-end; user interaction

Please use the following format when citing this chapter:

Attilio, F., Di Nunzio, P., Di Gregorio, F., and Meo, A.R., 2006, in IFIP International

Federation for Information Processing, Volume 203, Open Source Systems, eds. Damiani, E., Fitzgerald, B., Scacchi, W., Scotto, M., Succi, G., (Boston: Springer), pp. 337-338 
was poor and support for non-latin alphabets only partially working (mainly because of the limitations of a fixed-size cell-based output device.)

\section{The graphical debian-installer}

Classical text-based Debian installer supports 13 different architectures (from embedded systems to mainframes) and provides a solid technical base on which to build a graphical installer:

1. It is completely based on independent back-end modules (micro-debs or udebs) to which the install procedures (hardware recongition, hard disk partitioning, base system install, ...) are delegated.

2. The back-end modules communicates with the front-end using a welldefined and well-tested protocol (i.e., the debconf protocol.)

3. It is ready for localization and internationalization (even if the text-based front-end cannot render correctly non-latin or complex alphabets.)

4. Can be easily customized by providing package pre-configuration (preseeds) and/or custom procedures in the form of extra udebs.

The development of a graphical front-end module was based on previous work that used the GTK toolkit and was coordinated on the debian-boot mailing list, getting precious feedback and support by the official Debian Installer team. What we wanted was to be able to perform graphical installations even on low-resources machines, so we gave up using an $\mathrm{X}$ server and decided to put efforts in reviving the GTK-overDirectFrameBuffer project. DirectFrameBuffer (DFB) is a small set of libraries designed to be used in embedded Linux systems: the GTK-over-DFB project consists in a GDK backend module for the GTK libraries that allows GTK to run even without an X server. The work on the graphical debian-installer also gave new life to the DFB port of the GTK libraries: some talented developers from other projects put a great deal of efforts in fixing it and making it work with the last releases of GTK.

Hand-crafted prototypes of grapical ISO installations images led to full integration into the standard Debian ISO building system and after about 1 year of work the debian-installer team officially adopted the new codebase and announced the next Debian release will feature the new graphical front-end. The prototypes allows optimal rendering of over 70 different languages, included Indic ones, and run on less then 64 megabytes of RAM.

Also, Debian-derived LinEx distribution, developed in Extremadura and whose regional governament even sponsored a worksession on the grapgical installer, is going to be the first Debian derived distribution to offer a graphical, native, debianinstaller. 